\title{
Acceptability with general orderings
}

\author{
Danny De Schreye, Alexander Serebrenik \\ Department of Computer Science, K.U. Leuven \\ Celestijnenlaan 200A, B-3001, Heverlee, Belgium \\ Email: \{Danny.DeSchreye, Alexander.Serebrenik\}@cs.kuleuven.ac.be
}

\begin{abstract}
We present a new approach to termination analysis of logic programs. The essence of the approach is that we make use of general orderings (instead of level mappings), like it is done in transformational approaches to logic program termination analysis, but we apply these orderings directly to the logic program and not to the term-rewrite system obtained through some transformation. We define some variants of acceptability, based on general orderings, and show how they are equivalent to LD-termination. We develop a demand driven, constraint-based approach to verify these acceptability-variants.

The advantage of the approach over standard acceptability is that in some cases, where complex level mappings are needed, fairly simple orderings may be easily generated. The advantage over transformational approaches is that it avoids the transformation step all together.
\end{abstract}

Keywords: termination analysis, acceptability, orderings.

\section{Introduction}

It is not uncommon in research to have different research communities that tackle a same problem from a very different perspective or using totally different techniques. In some cases, such communities may co-exist for many years without much integration, cross-fertilisation or even decent comparison of the relative merits and drawbacks of competing approaches.

In the context of termination analysis of logic programs, two such subcommunities are those who develop and apply "transformational" approaches and those working on "direct" ones. A transformational approach first transforms the logic program into an "equivalent" term-rewrite system (or, in some cases, into an equivalent functional program). Here, equivalence means that, at the very least, the termination of the term-rewrite system should imply the termination of the logic program, for some predefined collection of queries ${ }^{1}$. Direct approaches do not include such a transformation, but prove the termination directly on the basis of the logic program.

Besides the transformation step itself, there is one other technical difference between these approaches. Direct approaches usually prove termination on the

\footnotetext{
${ }^{1}$ The approach of Arts [5] is exceptional in the sense that the termination of the logic program is concluded from a weaker property of single-redex normalisation of the term-rewrite system.
} 
basis of a well-founded ordering over the natural numbers. More specifically, they use a level mapping, which maps atoms to natural numbers, and, they verify appropriate decreases of this level mapping on the atoms occurring in the clauses. On the other hand, transformational approaches make use of more general well-founded orderings over terms, such as reduction orderings, or more specifically simplification orderings, or others (see [14]).

At least for the direct approaches the systematic choice for level mappings and norms - functions which map each term (module variable renaming) to a corresponding natural number-instead of general orderings, seems arbitrary and ad hoc. More generally, the relative merits and drawbacks of these two lines of work are not well understood. This has been the main motivation for this paper. We present an initial study on the use of general well-founded orderings as a means of directly proving the termination of logic programs - without intermediate transformation. In particular,

- we study whether the theoretical results on acceptability can be reformulated on the basis of general orderings,

- we evaluate to what extent the use of the general orderings (instead of level mappings) either improves or deteriorates the direct approaches.

To illustrate the latter point, consider the following program, that formulates some of the rules for computing the repeated derivative of a linear function in one variable $u$ (see also [16]) :

Example 1.

$$
\begin{aligned}
& d(\operatorname{der}(u), 1) . \\
& d(\operatorname{der}(A), 0) \leftarrow \text { number }(A) . \\
& d(\operatorname{der}(X+Y), D X+D Y) \leftarrow d(\operatorname{der}(X), D X), d(\operatorname{der}(Y), D Y) . \\
& d(\operatorname{der}(X * Y), X * D Y+Y * D X) \leftarrow d(\operatorname{der}(X), D X), d(\operatorname{der}(Y), D Y) . \\
& d(\operatorname{der}(\operatorname{der}(X)), D D X) \leftarrow d(\operatorname{der}(X), D X), d(\operatorname{der}(D X), D D X) .
\end{aligned}
$$

We are interested in proving LD-termination, i.e., finiteness of the SLD-tree constructed using the left-to-right selection rule of Prolog, of the program above together with the queries of the form $d(t, v)$, where $t$ is a term, expressing a derivative of a linear function in one variable $u, \operatorname{such}$ as $\operatorname{der}(\operatorname{der}(u * u * u+3 *$ $u * u+3 * u+1)$ ), and $v$ is a fresh variable, that will be unified with the result of the computation.

Doing this on the basis of a level-mapping is hard. For this example, a levelmapping that decreases between two sequential calls of $d$ is a non-linear function. In particular, a level mapping $|\cdot|$, and a norm $\|\cdot\|$, such that: $|d(X, Y)|=\|X\|$, $|\operatorname{number}(X)|=0,\|\operatorname{der}(X)\|=2^{\|X\|},\|X+Y\|=\max (\|X\|,\|Y\|)+1,\|X * Y\|=$ $\max (\|X\|,\|Y\|)+1,\|u\|=2,\|n\|=2$, if $n$ is a number,would be needed. No automatic system for proving termination on the basis of level mappings is able to generate such mappings. Moreover, we believe, that it would be very difficult to extend existing systems to support generation of appropriate nonlinear mappings. 
Although we have not yet presented our general-well-founded ordering approach, it should be intuitively clear, that we can capture the decrease in ordering between the $\operatorname{der}(X)$ and $D X$ by using an ordering on terms that gives the highest "priority" to the functor der.

On the other hand, using level mappings and norms allows sometimes to explore more precise information on atoms and terms, that cannot be expressed by general orderings, such as arithmetical relations between terms. This information can sometimes be crucial in proving termination as the following program from $[10,13]$ demonstrates.

Example 2.

$$
\begin{aligned}
& \operatorname{conf}(X) \leftarrow \operatorname{delete}_{2}(X, Z), \operatorname{delete}(U, Y, Z), \operatorname{conf}(Y) . \\
& \operatorname{delete}_{2}(X, Y) \leftarrow \operatorname{delete}(U, X, Z), \operatorname{delete}(V, Z, Y) . \\
& \operatorname{delete}(X,[X \mid T], T) . \\
& \operatorname{delete}(X,[H \mid T],[H \mid T 1]) \leftarrow \operatorname{delete}(X, T, T 1) .
\end{aligned}
$$

Note that by reasoning in terms of sizes of terms, we can infer that the size decreases by 2 after the call to delete 2 predicate in the first clause and then increases by 1 in the subsequent call to the delete predicate. In total, sizes allow us to conclude a decrease. Reasoning in terms of ordering relations only, however, does not allow to conclude the overall decrease from the facts that the third argument of delete predicate is smaller (with respect to some $>$ ) than the second one and that the first argument of delete 2 predicate is greater (with respect to $>$ ) than the second one.

As can be expected, theoretically both approaches are essentially equivalent. We will introduce a variant of the notion of acceptability, based on general orderings, which is again equivalent to termination in a similar way as in the level mapping based approach. On the more practical level, as illustrated in the two examples above, neither of the approaches is strictly better: the general orderings provide a larger set of orderings to select from (in particular, note that orderings based on level mappings and norms are general orderings), the level mapping approach provides arithmetic, on top of mere ordering.

In the remainder of this paper, we will start off from a variant of the notion of acceptability with respect to a set, as introduced in [11], obtained by replacing level mappings by orderings. We show how this variant of acceptability remains equivalent to termination under the left-to-right selection rule, for certain goals. Then, we illustrate how this result can be used to prove termination with some examples. We also provide a variant of the acceptability condition, as introduced in [4], and discuss advantages and disadvantages of each approach. Next, we discuss automation of the approach. We elaborate on a demand-driven method to set-up and verify sufficient preconditions for termination. In this method, the aim is to derive - in, as much as possible, a constructive way - a well-founded ordering over the set of all atoms and terms of the language underlying the program, that satisfies the termination condition. 


\section{Preliminaries}

\subsection{Logic Programs}

We follow the standard notation for terms and atoms. A query is a finite sequence of atoms. Given an atom $A, \operatorname{rel}(A)$ denotes the predicate occurring in $A$. Term $_{P}$ and Atom $_{P}$ denote, respectively, sets of all terms and atoms that can be constructed from the language underlying $P$. The extended Herbrand Universe $U_{P}^{E}$ (the extended Herbrand base $B_{P}^{E}$ ) is a quotient set of $\operatorname{Term}_{P}\left(\right.$ Atom $\left._{P}\right)$ modulo the variant relation.

We refer to an SLD-tree constructed using the left-to-right selection rule of Prolog, as an LD-tree. We will say that a goal $G L D$-terminates for a program $P$, if the LD-tree for $(P, G)$ is finite.

The following definition is borrowed from [2].

Definition 1. Let $P$ be a program and $p, q$ be predicates occurring in it.

- We say that $p$ refers to $q$ in $P$ if there is a clause in $P$ that uses $p$ in its head and $q$ in its body.

- We say that $p$ depends on $q$ in $P$ and write $p \sqsupseteq q$, if $(p, q)$ is in the transitive, reflexive closure of the relation refers to.

- We say that $p$ and $q$ are mutually recursive and write $p \simeq q$, if $p \sqsupseteq q$ and $q \sqsupseteq p$.

\subsection{Quasi-orderings and orderings}

A quasi-ordering over a set $S$ is a reflexive and transitive relation $\geq$ defined on elements of $S$. We define the associated equivalence relation $\leq \geq$ as $s \leq \geq t$ if and only if $s \geq t$ and $t \geq s$, and the associated ordering $>$ as $s>t$ if and only if $s \geq t$ but not $t \geq s$. If neither $s \geq t$, nor $t \geq s$ we write $s \|_{>} t$. Sometimes, in order to distinguish between different quasi-orderings and associated relations we also use $\succeq, \succ, \preceq \succeq$ and $\|_{\succ}$.

An ordered set $S$ is said to be well-founded if there are no infinite descending sequences $s_{1}>s_{2}>\ldots$ of elements of $S$. If the set $S$ is clear from the context we will say that the ordering, defined on it, is well-founded. We'll also say that a quasi-ordering is well-founded if the ordering associated with it, is well-founded.

Definition 2. Let $\geq$ be a quasi-ordering on a set $T$. A quasi-ordering $\succeq$ defined on a set $S \supseteq T$ is called a proper extension of $\geq$ if

- $t_{1} \geq t_{2}$ implies $t_{1} \succeq t_{2}$ for all $t_{1}, t_{2} \in T$.

- $t_{1}>t_{2}$ implies $t_{1} \succ t_{2}$ for all $t_{1}, t_{2} \in T$.

The study of termination of term-rewriting systems caused intensive study of orderings on terms. A number of useful properties were established.

Definition 3. Let $>$ be an ordering on $U_{P}^{E} \cup B_{P}^{E}$. 
$->$ is called monotonic if $s_{1}>s_{2}$ implies $f\left(\bar{t}_{1}, s_{1}, \bar{t}_{2}\right)>f\left(\bar{t}_{1}, s_{2}, \bar{t}_{2}\right)$ and $p\left(\bar{t}_{1}, s_{1}, \bar{t}_{2}\right)>p\left(\bar{t}_{1}, s_{2}, \bar{t}_{2}\right)$ for any terms $s_{1}$ and $s_{2}$, sequences of terms $\bar{t}_{1}$ and $\bar{t}_{2}$, function symbol $f$ and predicate $p$.

$->$ is said to have the subterm property if $f\left(\bar{t}_{1}, s, \bar{t}_{2}\right)>s$ holds for any term $f\left(\bar{t}_{1}, s, \bar{t}_{2}\right)$.

We extend the definition above to quasi-orderings.

Definition 4. Let $\geq$ be a quasi-ordering on terms.

$-\geq$ is called monotonic if

- $s_{1} \geq s_{2}$ implies $f\left(\bar{t}_{1}, s_{1}, \bar{t}_{2}\right) \geq f\left(\bar{t}_{1}, s_{2}, \bar{t}_{2}\right)$ and $p\left(\bar{t}_{1}, s_{1}, \bar{t}_{2}\right) \geq p\left(\bar{t}_{1}, s_{2}, \bar{t}_{2}\right)$ for any terms $s_{1}$ and $s_{2}$, sequences of terms $\bar{t}_{1}$ and $\bar{t}_{2}$, function symbol $f$ and predicate $p$ and

- the associated ordering is monotonic.

$-\geq$ is said to have the subterm property if the associated ordering has the subterm property.

The following are examples of orderings: $>$ on the set of numbers, lexicographic ordering on the set of strings (this is the way the entries are ordered in dictionaries), multiset ordering and recursive path ordering [14]. The following are examples of quasi-orderings: $\geq$ on the set of numbers, $\supseteq$ on the power set of some set.

For our purposes monotonicity and subterm properties are too restrictive. Thus, we assign to each predicate or functor a subset of argument positions, such that for the argument positions in this subset the specified properties hold. We will say that a predicate $p$ (a functor $f$ ) is monotone (has a subterm property) on a specified subset of argument positions. The formal study of these weaker notions may be found in [27].

Example 3. Let $f$ be a functor of arity two, and $a, b$ two terms, such that $a>b$. Let $f$ be monotone in the first argument position. Then, $f(a, c)>f(b, c)$ holds for any term $c$, but there might be some term $c$, such that $f(c, a) \ngtr f(c, b)$.

\section{Order-acceptability with respect to a set}

In this section we present and discuss some of the theory we developed to extend acceptability to general orderings. In the literature, there are different variants of acceptability. The most well-known of these is the acceptability as introduced by Apt and Pedreschi [4]. This version is defined and verified on the level of ground instances of clauses, but draws its practical power mostly from the fact that termination is proved for any bounded goal. Here, boundedness is a notion related to the selected level mapping and requires that the set $\{|G \theta| \quad \mid \quad \theta$ is a grounding substitution for goal $G\}$ is bounded in the natural numbers, where $|\cdot|: B_{P} \rightarrow \mathcal{N}$ denotes the level mapping.

Another notion of acceptability is the "acceptability with respect to a set of goals", introduced in [11]. This notion allows to prove termination with respect 
to any set of goals of interest. However, it relies on procedural concepts, such as calls and computed answer substitution. It was designed to be verified through global analysis, for instance through abstract interpretation.

A variant of acceptability with respect to a set that avoids the drawbacks of using procedural notions and that can be verified on a local level was designed in [13]. This variant required that the goals of interest are rigid under the given level mapping. Here, rigidity means that $|G \theta|=|G|$, for any substitution $\theta$, where $|\cdot|: B_{P}^{E} \rightarrow \mathcal{N}$ now denotes a generalised level mapping, defined on the extended Herbrand base.

Comparing the notions of boundedness and rigidity in the context of a level mapping based approach, it is clear that boundedness is more general than rigidity. If the level mapping of a goal is invariant under substitution, then the level mapping is bounded on the set of instances of the goal, but not conversely.

Given the latter observation and given that acceptability of [4] is a more generally known and accepted notion, we started our work by generalising this variant.

However, it turned out that generalising the concept of boundedness to general orderings proved to be very difficult. We postpone the discussion on this issue until after we formulated the results, but because of these complications, we only arrived at generalised acceptability conditions that are useful in the context of well-moded and simply moded programs and goals.

Because of this, we then turned our attention to acceptability with respect to a set. Here, the generalisation of rigidity was less complicated, so that in the end we obtained the strongest results for this variant of acceptability. Therefore, we first present order-acceptability with respect to a set of goals. We need the following notion.

Definition 5. [12] Let $P$ be a definite program and $S$ be a set of atomic queries. The call set, $C a l l(P, S)$, is the set of all atoms $A$, such that a variant of $A$ is a selected atom in some derivation for $P \cup\{\leftarrow Q\}$, for some $Q \in S$ and under the left-to-right selection rule.

To illustrate this definition recall the following example $[2,13]$.

Example 4.

$$
\begin{aligned}
& \text { permute }([],[]) . \\
& \operatorname{permute}(L,[E l \mid T]) \leftarrow \operatorname{delete}(E l, L, L 1), \operatorname{permute}(L 1, T) . \\
& \operatorname{delete}(X,[X \mid T], T) . \\
& \operatorname{delete}(X,[H \mid T],[H \mid T 1]) \leftarrow \operatorname{delete}(X, T, T 1) .
\end{aligned}
$$

Let $S$ be $\left\{\right.$ permute $\left(t_{1}, t_{2}\right) \mid t_{1}$ is a nil-terminated list and $t_{2}$ is a free variable . Then, $\operatorname{Call}(P, S)=$

$$
S \cup\left\{\text { delete }\left(t_{1}, t_{2}, t_{3}\right) \mid t_{1}, t_{3} \text { are free variables and } t_{2} \text { is a nil-terminated list }\right\} \text {. }
$$

Such information about $S$ could for instance be expressed in terms of the rigid types of Janssens and Bruynooghe $[21]$ and $C a l l(P, S)$ could be computed using the type inference of [21]. 
The following definition generalises the notion of acceptability with respect to a set [12] in two ways: 1) it generalises it to general quasi-orderings, 2) it generalises it to mutual recursion, using the standard notion of mutual recursion [2].

Definition 6. Let $S$ be a set of atomic queries and $P$ a definite program. $P$ is order-acceptable with respect to $S$ if there exists a well-founded quasi-ordering $\geq$, such that

- for any $A \in \operatorname{Call}(P, S)$

- for any clause $A^{\prime} \leftarrow B_{1}, \ldots, B_{n}$ in $P$, such that $\operatorname{mgu}\left(A, A^{\prime}\right)=\theta$ exists,

- for any atom $B_{i}$, such that $\operatorname{rel}\left(B_{i}\right) \simeq \operatorname{rel}(A)$

- for any computed answer substitution $\sigma$ for $\leftarrow\left(B_{1}, \ldots, B_{i-1}\right) \theta$ :

$$
A>B_{i} \theta \sigma
$$

The following establishes the connection between order-acceptability with respect to a set $S$ and LD-termination for queries in $S$.

Theorem 1. Let $P$ be a program. $P$ is order-acceptable with respect to a set of atomic queries $S$ if and only if $P$ is LD-terminating for all queries in $S$.

Proof. For all proofs we refer to [27].

We postpone applying the Theorem 1 to Example 4 until a more syntactic way of verifying order-acceptability with respect to a set is developed.

To do this, we extend the sufficient condition of [13], that imposes the additional requirement of rigidity of the level mapping on the call set, to the case of general quasi-orderings.

First we adapt the notion of rigidity to general orderings.

Definition 7. (see also [8]) The term or atom $A \in U_{P}^{E} \cup B_{P}^{E}$ is called rigid with respect to a quasi-ordering $\geq$ if for any substitution $\theta, A \leq \geq A \theta$. In this case $\geq$ is said to be rigid on $A$.

The notion of the rigidity on a term (an atom) is naturally extended to the notion of rigidity on a set of atoms (terms). In particular, we will be interested in quasi-orderings that are rigid on $\operatorname{Call}(P, S)$ for some $P$ and $S$.

We also need interargument relations based on general orderings.

Definition 8. Let $P$ be a definite program, $p$ a predicate in $P$ with arity $n$. An interargument relation is a relation $R_{p} \subseteq\left\{p\left(t_{1}, \ldots, t_{n}\right) \mid t_{i} \in \operatorname{Term}_{P}\right\} . R_{p}$ is a valid interargument relation for $p$ if and only if for every $p\left(t_{1}, \ldots, t_{n}\right) \in$ $\operatorname{Atom}_{P}:$ if $P \models p\left(t_{1}, \ldots, t_{n}\right)$ then $p\left(t_{1}, \ldots, t_{n}\right) \in R_{p}$.

Usually, the interargument relation will be defined based on a quasi-ordering used for proving termination. However, in general, this need not be the case.

Example 5. Consider the following program.

$$
\begin{aligned}
& p(0,[]) . \\
& p(f(X),[X \mid T]) \leftarrow p(X, T) .
\end{aligned}
$$


The following interargument relations can be considered for $p:\left\{p\left(t_{1}, t_{2}\right) \mid\right.$ $\left.t_{2}>t_{1} \vee t_{1} \leq \geq t_{2}\right\}$, valid if $\geq$ is a quasi-ordering imposed by a list-length norm, $\|\cdot\|_{l}$. Recall, that for lists $\left\|\left[t_{1} \mid t_{2}\right]\right\|_{l}=1+\left\|t_{2}\right\|_{l}$, while the list-length of other terms is considered to be 0 . On the other hand, $\left\{p\left(t_{1}, t_{2}\right) \mid t_{1}>t_{2} \vee t_{1} \leq \geq t_{2}\right\}$ is valid, if $\geq$ is a quasi-ordering imposed by a term-size norm.

Using general (non-norm based) quasi-orderings, $\left\{p\left(t_{1}, t_{2}\right) \mid t_{1}>t_{2}\right\}$ is valid, for example, for the recursive path ordering [14] with the following ordering on functors: $f / 1 \succ . / 2$, where.$/ 2$ is a function symbol defining lists, and $0 \succ[$. Alternatively, $\left\{p\left(t_{1}, t_{2}\right) \mid t_{2}>t_{1}\right\}$ is valid, for example, for the recursive path ordering with the following ordering on functors: $. / 2 \succ f / 1$ and [] $\succ 0$.

Using the notion of rigidity we state a sufficient condition for order-acceptability with respect to a set.

Theorem 2. (rigid order-acceptability with respect to $S$ ) Let $S$ be a set of atomic queries and $P$ be a definite program. Let $\geq$ be a quasi-ordering on $U_{P}^{E}$ and for each predicate $p$ in $P$, let $R_{p}$ be a valid interargument relation for $p$. If there exists a well-founded proper extension $\succeq$ of $\geq$ to $U_{P}^{E} \cup B_{P}^{E}$, which is rigid on Call $(P, S)$ such that

- for any clause $H \leftarrow B_{1}, \ldots, B_{n} \in P$, and

- for any atom $B_{i}$ in its body, such that $\operatorname{rel}\left(B_{i}\right) \simeq \operatorname{rel}(H)$,

- for any substitution $\theta$, such that the arguments of the atoms in $\left(B_{1}, \ldots, B_{i-1}\right) \theta$ all satisfy their associated interargument relations $R_{\text {rel }\left(B_{1}\right)}, \ldots, R_{\text {rel }\left(B_{i-1}\right)}$

$$
H \theta \succ B_{i} \theta
$$

then $P$ is order-acceptable with respect to $S$.

The stated condition is sufficient for order-acceptability, but is not necessary for it. Indeed, consider the following example:

Example 6 .

$$
\begin{aligned}
& p(X) \leftarrow q(X, Y), p(Y) . \\
& q(a, b) .
\end{aligned}
$$

Query $\leftarrow p(X)$ terminates with respect to this program. Thus, Theorem 1 implies the program is order-acceptable with respect to $\{p(X)\}$. However, the conditions of Theorem 2 do not hold. If $\geq$ is a quasi-ordering that satisfies these conditions, then $p(a) \leq \geq p(b)$ is implied by rigidity and $p(a)>p(b)$ is implied by the decrease, contradicting the definition of $>$.

We continue the analysis of Example 4 and show how Theorem 2 is used.

Example 7. Let $\succeq$ be a well-founded quasi-ordering on $U_{P}^{E} \cup B_{P}^{E}$, such that:

- for all terms $t_{1}, t_{21}$ and $t_{22}:$ permute $\left(t_{1}, t_{21}\right) \preceq \succeq$ permute $\left(t_{1}, t_{22}\right)$. 
- for all terms $t_{11}, t_{12}, t_{2}, t_{31}, t_{32}: \operatorname{delete}\left(t_{11}, t_{2}, t_{31}\right) \preceq \succeq \operatorname{delete}\left(t_{12}, t_{2}, t_{32}\right)$.

- for all terms $t_{11}, t_{12}$ and $t_{2}:\left[t_{11} \mid t_{2}\right] \preceq \succeq\left[t_{12} \mid t_{2}\right]$.

That is, we impose that the quasi-ordering is invariant on predicate argument positions and functor argument positions that may occur with a free variable in Call $(P, S)$. Furthermore, we impose that $\succeq$ has the subterm and monotonicity properties at all remaining predicate or functor argument positions.

First we investigate the rigidity of $\succeq$ on $\operatorname{Call}(P, S)$, namely: $G \theta \preceq \succeq G$ for any $G \in \operatorname{Call}(P, S)$ and any $\theta$. Now any effect that the application of $\theta$ to $G$ may have on $G$ needs to be through the occurrence of some variable in $G$. However, because we imposed that $\succeq$ is invariant on all predicate and functor argument positions that may possibly contain a variable in some call, $G \theta \preceq \succeq G$.

Associate with delete the interargument relation $R_{\text {delete }}=\left\{\right.$ delete $\left(t_{1}, t_{2}, t_{3}\right) \mid$ $\left.t_{2} \succ t_{3}\right\}$. First, we verify that this interargument relationship is valid. Note, that an interargument relationship is valid whenever it is a model for its predicate. Thus, to check whether $R_{\text {delete }}$ is valid, $T_{P}\left(R_{\text {delete }}\right) \subseteq R_{\text {delete }}$ is checked. For the non-recursive clause of delete the inclusion follows from the subset property of $\succeq$, while for the recursive one, from the monotonicity of it.

Then, consider the recursive clauses of the program.

- permute. If delete $(E l, L, L 1) \theta$ satisfies $R_{\text {delete }}$, then $L \theta \succ L 1 \theta$. By the monotonicity, permute $(L, T) \theta \succ$ permute $(L 1, T) \theta$. By the property stated above, permute $(L,[E l \mid T]) \theta \preceq \succeq$ permute $(L, T) \theta$. Thus, the desired decrease permute $(L,[E l \mid T]) \theta \succ$ permute $(L 1, T) \theta$ holds.

- delete. By the properties of $\succ$ stated above: $\operatorname{delete}(X,[H \mid T],[H \mid T 1]) \succ$ $\operatorname{delete}(X, T,[H \mid T 1])$ and $\operatorname{delete}(X, T,[H \mid T 1]) \preceq \succeq \operatorname{delete}(X, T, T 1)$. Thus, $\operatorname{delete}(X,[H \mid T],[H \mid T 1]) \succ \operatorname{delete}(X, T, T 1)$.

We have shown that all the conditions of Theorem 2 are satisfied, and thus, $P$ is order-acceptable with respect to $S$. By Theorem $1, P$ terminates for all queries in $S$.

Observe, that we do not need to construct the actual ordering, but only to prove that there is one, that meets all the requirements posed. In this specific case, the requirement of subterm and monotonicity on the remaining argument positions is satisfiable.

\section{The results for acceptability with respect to a model}

In this section we briefly discuss some of the results we obtained in generalising the acceptability notion of $[4,17]$. Since these results are weaker than those presented in the previous section, we do not elaborate on them in full detail.

For a predicate $p$ with arity $n$, a mode is an atom $p\left(m_{1}, \ldots, m_{n}\right)$, where $m_{i} \in\{$ in, out $\}$ for $1 \leq i \leq n$. Positions with in are called input positions, and positions with out are called output positions of $p$. We assume that a fixed mode is associated with each predicate in a program. To simplify the notation, an atom written as $p(\mathbf{s}, \mathbf{t})$ means: $\mathbf{s}$ is the vector of terms filling the input positions, and 
$\mathbf{t}$ is the vector of terms filling the output positions. Furthermore, by $\operatorname{Var}(\mathbf{s})$ we denote the set of variables occuring in vector of terms $\mathbf{s}$ [2].

Below, we assume that modes for the program and goal are given. For any atom $A$ and a mode $m_{A}$ for $A$, we denote by $A^{\text {inp }}$ the atom obtained from $A$ by removing all output arguments. E.g., let $A=p(f(2), 3, X)$ and $m_{A}=$ $p($ in, in, out $)$, then $A^{\text {inp }}=p(f(2), 3)$.

Definition 9. Let $\geq$ be a quasi-ordering relation on $B_{P}^{E}$. We say that $\geq$ is output-independent if for any two moded atoms $A$ and $B: A^{\text {inp }}=B^{\text {inp }}$ implies $A \leq \geq B$.

The first class of the programs we consider, are well-moded programs.

Definition 10. [2]

1. A query $p_{1}\left(\mathbf{s}_{\mathbf{1}}, \mathbf{t}_{\mathbf{1}}\right), \ldots, p_{n}\left(\mathbf{s}_{\mathbf{n}}, \mathbf{t}_{\mathbf{n}}\right)$ is called well-moded if for $i \in[1, n]$

$$
\operatorname{Var}\left(\mathbf{s}_{\mathbf{i}}\right) \subseteq \bigcup_{j=1}^{i-1} \operatorname{Var}\left(\mathbf{t}_{\mathbf{j}}\right)
$$

2. A clause $p_{0}\left(\mathbf{t}_{\mathbf{0}}, \mathbf{s}_{\mathbf{n}+\mathbf{1}}\right) \leftarrow p_{1}\left(\mathbf{s}_{\mathbf{1}}, \mathbf{t}_{\mathbf{1}}\right), \ldots, p_{n}\left(\mathbf{s}_{\mathbf{n}}, \mathbf{t}_{\mathbf{n}}\right)$ is called well-moded if for $i \in[1, n+1]$

$$
\operatorname{Var}\left(\mathbf{s}_{\mathbf{i}}\right) \subseteq \bigcup_{j=0}^{i-1} \operatorname{Var}\left(\mathbf{t}_{\mathbf{j}}\right)
$$

3. A program is called well-moded if every clause of it is.

For well-moded programs, order-acceptability in the style of [4] can now be defined as follows.

Definition 11. Let $P$ be a well-moded program, $\geq$ an output-independent wellfounded quasi-ordering and $I$ a model for $P$. The program $P$ is called orderacceptable with respect to $\geq$ and $I$ if for all $A \leftarrow B_{1}, \ldots, B_{n}$ in $P$ and all substitutions $\theta$, such that $(A \theta)^{\text {inp }}$ and $B_{1} \theta, \ldots, B_{i-1} \theta$ are ground and $I \models B_{1} \theta \wedge$ $\ldots \wedge B_{i-1} \theta$ holds: $A \theta>B_{i} \theta$.

$P$ is called order-acceptable if it is order-acceptable with respect to some output-independent well-founded quasi-ordering and some model. Note the similarity and the difference with the notion of well-acceptability introduced by Etalle, Bossi and Cocco [17] — both notions relay on "ignoring" the output positions. However, the approach suggested in [17] measures atoms by level-mappings, while our approach is based on general orderings. In addition [17] requires a decrease only between atoms of mutually recursive predicates. Similarly, one might use the notion of order-acceptability that requires a decrease only between atoms of mutually recursive predicates. This definition will be equivalent to the one we used, since for atoms of non-mutually recursive predicates the dependency relation, $\sqsupset$, can always be used to define an ordering. Since every level mapping 
naturally gives rise to the ordering on atoms, that is $A_{1} \succ A_{2}$ if $\left|A_{1}\right|>\left|A_{2}\right|$, we conclude that every well-acceptable program is order-acceptable.

The following theorem states that order-acceptability of a well-moded program is sufficient for termination of well-moded goals with respect to this program. Etalle, Bossi and Cocco [17] call such a program well-terminating.

Theorem 3. Let $P$ be a well-moded program, that is order-acceptable with respect to an output-independent well-founded quasi-ordering $\geq$ and a model I. Let $G$ be a well-moded goal, then $G$ LD-terminates.

Note that if the requirement of well-modedness of the program $P$ is dropped then the theorem no longer holds.

Example 8.

$$
\begin{aligned}
& p(a) \leftarrow q(X) . \\
& q(f(X)) \leftarrow q(X) .
\end{aligned}
$$

We assume the modes $p($ in) and $q($ in $)$ to be given. This program is not wellmoded with respect to the given modes, because $p(a)$ calls $q / 1$ with a free variable, but it satisfies the remaining conditions of order-acceptability with respect to the following quasi-ordering $\geq$ on terms $p(a)>q(t)$ and $q(f(t))>q(t)$ for any term $t$ and $t \leq \geq s$ only if $t$ and $s$ are syntactically identical, and the following model $I=\{p(a), q(a), q(f(a)), q(f(f(a))), \ldots\}$. However, note that the well-moded goal $p(a)$ is non-terminating.

Unfortunately, well-modedness is not sufficient to make the converse to hold. That is, there is a well-moded program $P$ and a well-moded goal $G$, such that $G$ is LD-terminating with respect to $P$, but $P$ is not order-acceptable.

Example 9. Consider the following program

$$
p(f(X)) \leftarrow p(g(X)) .
$$

with the mode $p($ out $)$. This program is well-moded, the well-moded goal $p(X)$ terminates with respect to this program, but it is not order-acceptable, since the required decrease $p(f(X))>p(g(X))$ violates output-independence of $\geq$.

Intuitively, the problem in the example occured, because some information has been passed via the output positions, i.e, $P$ is not simply moded.

Definition 12. [3]

1. A query $p_{1}\left(\mathbf{s}_{\mathbf{1}}, \mathbf{t}_{\mathbf{1}}\right), \ldots, p_{n}\left(\mathbf{s}_{\mathbf{n}}, \mathbf{t}_{\mathbf{n}}\right)$ is called simply moded if $\mathbf{t}_{\mathbf{1}}, \ldots, \mathbf{t}_{\mathbf{n}}$ is a linear family of variables and for $i \in[1, n]$

$$
\operatorname{Var}\left(\mathbf{s}_{\mathbf{i}}\right) \cap\left(\bigcup_{j=i}^{n} \operatorname{Var}\left(\mathbf{t}_{\mathbf{j}}\right)\right)=\emptyset .
$$


2. A clause $p_{0}\left(\mathbf{s}_{\mathbf{0}}, \mathbf{t}_{\mathbf{o}}\right) \leftarrow p_{1}\left(\mathbf{s}_{\mathbf{1}}, \mathbf{t}_{\mathbf{1}}\right), \ldots, p_{n}\left(\mathbf{s}_{\mathbf{n}}, \mathbf{t}_{\mathbf{n}}\right)$ is called simply moded if $p_{1}\left(\mathbf{s}_{\mathbf{1}}, \mathbf{t}_{\mathbf{1}}\right), \ldots, p_{n}\left(\mathbf{s}_{\mathbf{n}}, \mathbf{t}_{\mathbf{n}}\right)$ is simply moded and

$$
\operatorname{Var}\left(\mathbf{s}_{\mathbf{0}}\right) \cap\left(\bigcup_{j=1}^{n} \operatorname{Var}\left(\mathbf{t}_{\mathbf{j}}\right)\right)=\emptyset .
$$

3. A program is called simply moded if every clause of it is.

Indeed, if $P$ is simply moded the second direction of the theorem holds as well. This was already observed in [17] in the context of well-acceptability and well-termination. The following is an immediate corollary to Theorem 5.1 in [17]. As that theorem states for well-moded simply moded programs, welltermination implies well-acceptability. Therefore, well-terminating programs are order-acceptable.

Corollary 1. Let $P$ be a well-moded simply moded program, LD-terminating for any well-moded goal. Then there exists a model $I$ and an output-independent well-founded quasi-ordering $\geq$, such that $P$ is order-acceptable with respect to $I$ and $\geq$.

To conclude, we briefly discuss why it is difficult to extend the notions of order-acceptability to the non well-moded case, using a notion of boundedness, as it was done for standard acceptability [4]. In acceptability based on level mappings, boundedness ensures that the level mapping of a (non-ground) goal can only increase up to some finite bound when the goal becomes more instantiated. Observe that every ground goal is trivially bounded.

The most naive approach to generalisation of boundedness is replacing comparisons of level mappings with orderings, that is defining an atom $A$ to be bounded with respect to an ordering $>$, if there exists an atom $C$ such that for all ground instances $A \theta$ of $A, C>A \theta$. Unfortunately, this definition is too week to impose termination.

Example 10.

$$
\begin{aligned}
& q \leftarrow p(X) . \\
& p(f(X)) \leftarrow p(X) . \\
& p(a) .
\end{aligned}
$$

Goal $p(X)$ is bounded with respect to the quasi-ordering such that $q>\ldots>$ $p(f(f(a)))>p(f(a))>p(a)$. Similarly, the decrease requirement between the head and the subgoals is satisfied, however the goal does not terminate.

Intuitively, the problem in this example occured due to the fact that infinitely many different atoms are smaller than the boundary. One can try to fix this problem by redefining boundedness as:

An atom $A$ is bounded with respect to an ordering $>$, if there exists an atom $C$ such that for all ground instances $A \theta$ of $A$ : $A \theta<C$, and $\left\{B \in B_{P}^{E} \mid B<C\right\}$ is finite. 
Such a definition imposes constraints which are very similar to the ones imposed by standard boundedness in the context of level mappings. However, one thing we loose is that it is no longer a generalisation of groundness. Consider an atom $p(a)$ and assume that our language contains a functor $f / 1$ and a constant $b$. Then one particular well-founded ordering is

$$
p(a)>\ldots>p(f(f(b)))>p(f(b))>p(b) .
$$

So, $p(a)$ is not bounded with respect to this ordering.

Because of such complications, we felt that the rigidity-based results of the previous section are the preferred generalisations to general orderings.

\section{$5 \quad$ A methodology for verifying order-acceptability}

In this section we present an approach leading towards automatic verification of the order-acceptability condition. The basic idea for the approach is inspired on the "constraint based" termination analysis proposed in [13]. We start off from the conditions imposed by order-acceptability, and systematically reduce these conditions to more explicit constraints on the objects of our search: the quasi-ordering $\geq$ and the interargument relations, $R_{p}$, or model $I$.

The approach presented below has been applied successfully to a number of examples that appear in the literature on termination, such as different versions of permute [6,22,13], dis-con [10], transitive closure [22], add-mult [25], combine, reverse, odd-even, at_least_double and normalisation [13], quicksort program [29, 2], derivative [16], distributive law [15], boolean ring [20], aiakl, bid [9], credit evaluation expert system [29], flatten [5], vanilla meta-interpreter solve [29] together with wide class of interpreted programs.

In the remainder of the paper, we explain the approach using some of these examples.

We start by showing how the analysis of Example 4, presented before, can be performed systematically. We stress the main steps of a methodology.

Example 11. $\geq$ should be rigid on $\operatorname{Call}(P, S)$. To enforce the rigidity, $\geq$ should ignore all argument positions in atoms in $\operatorname{Call}(P, S)$ that might be occupied by free variables, i.e., the second argument position of permute and the first and the third argument positions of delete. Moreover, since the first argument of permute and the second argument of delete are general nil-terminated lists, the first argument of.$/ 2$ should be ignored as well.

The decreases with respect to $>$ imposed in the order-acceptability with respect to a set $S$ are:

$$
\begin{gathered}
\operatorname{delete}(X,[H \mid T],[H \mid T 1]) \theta>\operatorname{delete}(X, T, T 1) \theta \\
\operatorname{delete}\left(E l, L, L_{1}\right) \theta \text { satisfies } R_{\text {delete }} \text { implies } \\
\operatorname{permute}(L,[E l \mid T]) \theta>\operatorname{permute}\left(L_{1}, T\right) \theta
\end{gathered}
$$


To express the rigidity constraints, we simplify each of these conditions by replacing the predicate argument positions that should be ignored by some arbitrary term-one of $v_{1}, v_{2}, \ldots$ The following conditions are obtained:

$$
\begin{gathered}
\operatorname{delete}\left(v_{1},[H \mid T] \theta, v_{2}\right)>\operatorname{delete}\left(v_{3}, T \theta, v_{4}\right) \\
\operatorname{delete}\left(E l, L, L_{1}\right) \theta \text { satisfies } R_{\text {delete }} \text { implies } \\
\quad \operatorname{permute}\left(L \theta, v_{1}\right)>\operatorname{permute}\left(L_{1} \theta, v_{2}\right)
\end{gathered}
$$

Observe that this replacement only partially deals with the requirements that the rigidity conditions expressed above impose: rigidity on functor arguments (the first argument of.$/ 2$ should be invariant with respect to the ordering) is not expressed. We keep track of such constraints implicitly, and only verify them at a later stage when additional constraints on the ordering are derived.

For each of the conditions (1) and (2), we have two options on how to enforce it:

Option 1): The decrease required in the condition can be achieved by imposing some property on $\geq$, which is consistent with the constraints that were already imposed on $\geq$ before.

In our example, condition (1) is satisfied by imposing the subterm property for the second argument of.$/ 2$ and monotonicity on the second argument of delete. The second argument of.$/ 2$ does not belong to a set of functor argument positions that should be ignored. Then, $\left[t_{1} \mid t_{2}\right]>t_{2}$ holds for any terms $t_{1}$ and $t_{2}$, and by the monotonicity of $>$ in the second argument of delete (1) holds.

In general we can select from a bunch of ordering properties, or even specific orderings, that were proposed in the literature.

Option 2): The required decrease is imposed as a constraint on the interargument relation(s) $R$ of the preceding atoms.

In the permute example, the decrease permute $(L \theta, t)>\operatorname{permute}\left(L_{1} \theta, t\right)$ cannot directly be achieved by imposing some constraint on $>$. Thus, we impose that the underlying decrease $L \theta>L_{1} \theta$ should hold for the intermediate body atoms (delete $\left(E l, L, L_{1}\right) \theta$ ) that satisfy the interargument relation $R_{\text {delete }}$.

Thus, in the example, the constraint is that $R_{\text {delete }}$ should be such that for all delete $\left(t_{1}, t_{2}, t_{3}\right)$ that satisfy $R_{\text {delete }} t_{2}>t_{3}$. As we have observed, the interargument relation is valid if it forms a model for its predicate. Thus, one way to constructively verify that a valid interargument relation $R_{\text {delete }}$ exists, such that the property $t_{2}>t_{3}$ holds for delete $\left(t_{1}, t_{2}, t_{3}\right)$ atoms is to simply impose that $M=\left\{\operatorname{delete}\left(t_{1}, t_{2}, t_{3}\right) \mid t_{2}>t_{3}\right\}$ itself is a model for the delete clauses in the program.

So our new constraint on $R_{\text {delete }}$ is that it should include $M$. Practically we can enforce this by imposing that $T_{P}(M) \subseteq M$ should hold. As shown in [27], this reduces to the constraints " $\left[t_{1} \mid t_{2}\right]>t_{2}$ " and " $t_{2}>t_{3}$ implies $\left[t \mid t_{2}\right]>\left[t \mid t_{3}\right]$ ". These are again fed into our Option 1) step, imposing a monotonicity property on the second argument of.$/ 2$ for $>$. At this point the proof is complete.

Recall that we do not need to construct actually the ordering, but only to prove that there is one, that meets all the requirements posed. 


\section{Further examples}

Although the simplicity of the permute example makes it a good choice to clarify our approach it does not well motivate the need for general orderings instead of level mappings. Indeed, it is well-known that permute can be dealt with using standard acceptability or acceptability with respect to a set [10].

In this section we provide a number of additional examples. Most of them (distributive law, derivative and solve) illustrate the added power of moving to general orderings. After these we present an alternative version of permute in order to discuss an extension of our approach that deals with interargument relations for conjunctions of (body-) atoms.

Before presenting the examples we recall once more the main steps of our approach. First, given a program $P$ and a set $S$ of goals, compute the set of calls Call $(P, S)$. Janssens and Bruynooghe [21] show how this can be done through abstract interpretation. Second, enforce the rigidity of $>$ on $C a l l(P, S)$, i.e., ignore all predicate or functor argument positions that might be occupied by free variables in $C a l l(P, S)$. Given the set of calls, this step can be performed in a completely automatic way. Third, repeatedly construct decreases with respect to $>$, such that the rigid order-acceptability condition will hold and check if those can be verified by some of the predefined orderings. While performing this verification step the trade-off between efficiency and power should be consideredusing more complex orderings may allow correct reasoning on more examples but might be computationally expensive.

First, we consider the distributive law program. This example originated from [15].

Example 12.

$$
\begin{aligned}
& \operatorname{dist}(x, x) . \\
& \operatorname{dist}(x * x, x * x) . \\
& \operatorname{dist}(X+Y, U+V) \leftarrow \operatorname{dist}(X, U), \operatorname{dist}(Y, V) . \\
& \operatorname{dist}(X *(Y+Z), T) \leftarrow \operatorname{dist}(X * Y+X * Z, T) . \\
& \operatorname{dist}((X+Y) * Z, T) \leftarrow \operatorname{dist}(X * Z+Y * Z, T) .
\end{aligned}
$$

Similarly to the repeated derivation example in the introduction, no linear norm is sufficient for proving termination. The simplest norm, we succeeded to find, providing a termination proof is the following one: $\|X * Y\|=\|X\| *\|Y\|$, $\|X+Y\|=\|X\|+\|Y\|+1,\|x\|=2$ and the level mapping is $|\operatorname{dist}(X, Y)|=\|X\|$. This norm cannot be generated automatically by termination analysers we are aware of.

In order to prove termination of a set of queries

$$
\left\{\operatorname{dist}\left(t_{1}, t_{2}\right) \mid t_{1} \text { is an expression in a variable } x \text { and } t_{2} \text { is a free variable }\right\}
$$

we use the rigid-acceptability condition. First the quasi-ordering, $\geq$, we are going to define should be rigid on a set of calls, i.e., it should ignore the second 
argument position of dist. Thus, in the decreases with respect to $>$ to follow we replace the second argument of dist with anonymous terms $v_{1}, v_{2}, \ldots$.

$$
\begin{aligned}
& \operatorname{dist}\left((X+Y) \theta, v_{1}\right)>\operatorname{dist}\left(X \theta, v_{2}\right) \\
& \operatorname{dist}(X, U) \theta \text { satisfies } R_{\text {dist }} \text { implies } \\
& \quad \operatorname{dist}\left((X+Y) \theta, v_{1}\right)>\operatorname{dist}\left(Y \theta, v_{2}\right) \\
& \operatorname{dist}\left((X *(Y+Z)) \theta, v_{1}\right)>\operatorname{dist}\left((X * Y+X * Z) \theta, v_{2}\right) \\
& \operatorname{dist}\left(((X+Y) * Z) \theta, v_{1}\right)>\operatorname{dist}\left((X * Z+Y * Z) \theta, v_{2}\right)
\end{aligned}
$$

The first two decreases are satisfied by any ordering having a subterm property for both arguments of $+/ 2$ and being monotonic with respect to the first argument position of dist. However, in order to satisfy the later two we need to use the recursive path ordering (rpo) [14], with $*$ preceding + with respect to an ordering on functors. If this ordering is used, the following holds for any $t_{1}, t_{2}$ and $t_{3}$ :

$$
\begin{aligned}
& t_{2}+t_{3}>t_{2} \\
& t_{1} *\left(t_{2}+t_{3}\right)>t_{1} * t_{2} \\
& t_{2}+t_{3}>t_{3} \\
& t_{1} *\left(t_{2}+t_{3}\right)>t_{1} * t_{3} \\
& t_{1} *\left(t_{2}+t_{3}\right)>t_{1} * t_{2}+t_{1} * t_{3} \quad \text { (using the properties of rpo) }
\end{aligned}
$$

This proves the third decrease with respect to $>$. The fourth one is proved analogously.

Now we can return to the motivating Example 1, on computing higher derivatives of polynomial functions in one variable.

\section{Example 13.}

$$
\begin{aligned}
& d(\operatorname{der}(u), 1) . \\
& d(\operatorname{der}(A), 0) \leftarrow \operatorname{number}(A) . \\
& d(\operatorname{der}(X+Y), D X+D Y) \leftarrow d(\operatorname{der}(X), D X), d(\operatorname{der}(Y), D Y) . \\
& d(\operatorname{der}(X * Y), X * D Y+Y * D X) \leftarrow d(\operatorname{der}(X), D X), d(\operatorname{der}(Y), D Y) . \\
& d(\operatorname{der}(\operatorname{der}(X)), D D X) \leftarrow d(\operatorname{der}(X), D X), d(\operatorname{der}(D X), D D X) .
\end{aligned}
$$

We are interested in proving termination of the queries that belong to the set $S=\left\{d\left(t_{1}, t_{2}\right) \mid t_{1}\right.$ is a repeated derivative of a function in a variable $u$ and $t_{2}$ is a free variable $\}$. So $S$ consists of atoms of the form $d(\operatorname{der}(u), X)$ or $d(\operatorname{der}(u *$ $u+u), Y)$ or $d(\operatorname{der}(\operatorname{der}(u+u)), Z)$, etc. Observe, that Call $(P, S)$ coincides with $S$.

We start by analysing the requirements that imposes the rigidity of $\geq$ on Call $(P, S)$. First, the second argument position of $d$ should be ignored, since it 
might be occupied by a free variable. Second, the first argument position of $d$ is occupied by a ground term. Thus, rigidity does not pose any restrictions on functors argument positions.

Then, we construct the decreases with respect to $>$ that follow from the rigid order-acceptability. The arguments that should be ignored are replaced by terms $v_{1}, v_{2}, \ldots$

$$
\begin{aligned}
& d\left(\operatorname{der}(X+Y) \theta, v_{1}\right)>d\left(\operatorname{der}(X) \theta, v_{2}\right) \\
& d(\operatorname{der}(X), D X) \theta \text { satisfies } R_{d} \text { implies } \\
& d\left(\operatorname{der}(X+Y) \theta, v_{1}\right)>d\left(\operatorname{der}(Y) \theta, v_{2}\right) \\
& d\left(\operatorname{der}(X * Y) \theta, v_{1}\right)>d\left(\operatorname{der}(X) \theta, v_{2}\right) \\
& d(\operatorname{der}(X), D X) \theta \text { satisfies } R_{d} \text { implies } \\
& \quad d\left(\operatorname{der}(X * Y) \theta, v_{1}\right)>d\left(\operatorname{der}(Y) \theta, v_{2}\right) \\
& d\left(\operatorname{der}(\operatorname{der}(X)) \theta, v_{1}\right)>d\left(\operatorname{der}(X) \theta, v_{2}\right) \\
& d(\operatorname{der}(X), D X) \theta \text { satisfies } R_{d} \operatorname{implies} \\
& \quad d\left(\operatorname{der}(\operatorname{der}(X)) \theta, v_{1}\right)>d\left(\operatorname{der}(D X) \theta, v_{2}\right)
\end{aligned}
$$

Conditions (3)-(7) impose monotonicity and subset properties to hold on the first argument of $d$. In order to satisfy condition (8), it is sufficient to prove that for any $\left(t_{1}, t_{2}\right) \in R_{d}$ holds that $t_{1}>t_{2}$. That is if $M=\left\{d\left(t_{1}, t_{2}\right) \mid t_{1}>t_{2}\right\}$ then $T_{P}(M) \subseteq M$. This may be reduced to the following conditions:

$$
\begin{aligned}
& \operatorname{der}(t)>1 \\
& t_{1} \in R_{\text {number implies }} \operatorname{der}\left(t_{1}\right)>0 \\
& \operatorname{der}\left(t_{1}\right)>t_{2} \& \operatorname{der}\left(t_{3}\right)>t_{4} \text { implies } \operatorname{der}\left(t_{1}+t_{3}\right)>t_{2}+t_{4} \\
& \operatorname{der}\left(t_{1}\right)>t_{2} \& \operatorname{der}\left(t_{3}\right)>t_{4} \text { implies } \operatorname{der}\left(t_{1} * t_{3}\right)>t_{1} * t_{4}+t_{2} * t_{3} \\
& \operatorname{der}\left(t_{1}\right)>t_{2} \& \operatorname{der}\left(t_{2}\right)>t_{3} \text { implies } \operatorname{der}\left(\operatorname{der}\left(t_{1}\right)\right)>t_{3}
\end{aligned}
$$

Condition (13) follows from monotonicity and transitivity of $>$. However, (10)(12) are not satisfied by general properties of $>$ and we need to specify the ordering. The ordering that meets these conditions is the recursive path ordering [14] with der having the highest priority.

As a next example we demonstrate that the suggested technique is useful for proving termination of meta-interpreters as well.

Example 14.

$$
\begin{aligned}
& \text { solve }(\text { true }) . \\
& \text { solve }((A, B)) \leftarrow \operatorname{solve}(A), \operatorname{solve}(B) . \\
& \text { solve }(A) \leftarrow \operatorname{clause}(A, B), \operatorname{solve}(B) .
\end{aligned}
$$

Even though the termination of an interpreted program might be easily proved with level-mappings, the termination proof of the meta-interpreter with respect to it cannot be immediately constructed based on the termination proof of the interpreted program. 
Indeed, let $P$ be the interpreted program:

$$
p([X, Y \mid T]) \leftarrow p([Y \mid T]), p(T) .
$$

Termination of the set of queries $\{p(t) \mid t$ is a list of a finite length $\}$ can be easily proved, for example by a using level mapping $|p(X)|=\|X\|_{l}$ and the listlength norm $\|\cdot\|_{l}$. However, when this program is considered together with this meta-interpreter these level-mapping and norm cannot be extended in a way allowing to prove termination, even though there exist a linear level-mapping and a linear norm that provide a termination proof. In the case of this example, the following linear level mapping is sufficient for proving termination:

$$
\begin{aligned}
& |\operatorname{solve}(A)|=\|A\| \\
& \|(A, B)\|=1+\|A\|+\|B\| \\
& \|p(X)\|=1+\|X\| \\
& \|[H \mid T]\|=1+3\|T\|
\end{aligned}
$$

The constraint-based approach of [13] is able to derive this level mapping. However, it cannot reuse any information from a termination proof of the interpreted program to do so, and the constraints set up for such examples are fairly complex ( $n$ body atoms are interpreted as a ,/2-term of depth $n$ and reasoning on them requires products of (at least) $n$ parameters). Most other approaches based on level mappings work on basis of fixed norms, like list-length and termsize, and therefore fail to prove termination of the example.

Applying general orderings allows to define a new ordering for the metainterpreter together with the interpreted program based on the ordering obtained for the interpreted program itself. More formally, given a quasi-ordering $\geq$, defined for the interpreted program above, define a quasi-ordering $\succeq$ on terms and atoms of the meta-interpreter, as follows (similarly to rpo [14]):

$-t \preceq \succeq s$ if one of the following holds:

- $t \leq \geq s$

- $t=\left(t_{1}, t_{2}\right), s=\left(s_{1}, s_{2}\right)$ and $t_{1} \preceq \succeq s_{1}, t_{2} \preceq \succeq s_{2}$

- $t=\operatorname{solve}\left(t_{1}\right), s=\operatorname{solve}\left(s_{1}\right)$ and $t_{1} \preceq \succeq s_{1}$

$-t \succ s$ if one of the following holds:

- $t>s$

- $t=f(\ldots), s=\left(s_{1}, s_{2}\right), f$ differs from,$/ 2$, solve $/ 1, t \succ s_{1}$ and $t \succ s_{2}$

- $t=\left(t_{1}, t_{2}\right)$ and either $t_{1} \succeq s$ or $t_{2} \succeq s$.

- $t=\operatorname{solve}\left(t_{1}\right), s=\operatorname{solve}\left(s_{1}\right)$ and $t_{1} \succ s_{1}$.

- $t=\operatorname{solve}\left(t_{1}\right), s=\operatorname{clause}\left(s_{1}, s_{2}\right)$

In our case $\geq$ is a list-length norm based ordering, and $\succeq$ is defined as specified. Then, $p([X, Y \mid T]) \succ(p([Y \mid T]), p(T))$. This provides the $\succ$-decrease for the second recursive clause of the meta-interpreter required in the rigid orderacceptability condition. Similarly, the decrease for the first recursive clause is provided by the subterm property that $\succ$ is defined to have, and thus, proving termination. 
By reasoning in a similar way, termination can be proved for the metainterpreter and wide class of interpreted programs: from the small examples, such as append and delete and up to bigger ones, like aiakl, bid [9], credit evaluation expert system [29], or even the distributive law program, presented in Example 12.

The previous examples do not illustrate our approach in full generality. In general, we may have clauses of the type

$$
p\left(t_{1}, \ldots, t_{n}\right) \leftarrow B_{1}, B_{2}, \ldots, B_{i-1}, q\left(s_{1}, \ldots, s_{m}\right), B_{i+1}, \ldots, B_{k} .
$$

where multiple intermediate body-atoms, $B_{1}, B_{2}, \ldots, B_{i-1}$ precede the (mutually) recursive body-atom $q\left(s_{1}, \ldots, s_{m}\right)$. In such cases the decrease with respect to $>$ between $p\left(t_{1}, \ldots, t_{n}\right) \theta$ and $q\left(s_{1}, \ldots, s_{m}\right) \theta$ required by the (rigid) order-

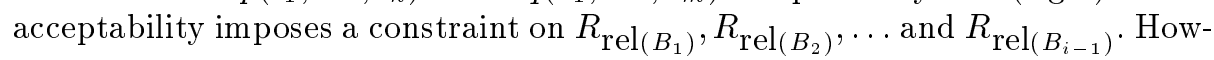
ever, our previous technique of using $T_{P}(M) \subseteq M$ to translate the required decrease to $R_{\operatorname{rel}\left(B_{1}\right)}, R_{\operatorname{rel}\left(B_{2}\right)}, \ldots, R_{\operatorname{rel}\left(B_{i-1}\right)}$ is not easily generalised. This is because several of the atoms $B_{1}, B_{2}, \ldots, B_{i-1}$ together may be responsible for the decrease and the $T_{P}(M) \subseteq M$ technique is not readily generalised to deal with multiple predicates.

One way to deal with this is based on early works on termination analysis $([31,25])$. Assume that the underlying decrease imposed by

$$
\begin{gathered}
B_{1} \theta, B_{2} \theta, \ldots, B_{i-1} \theta \text { satisfy } R_{\operatorname{rel}\left(B_{1}\right)}, R_{\operatorname{rel}\left(B_{2}\right)}, \ldots, R_{\operatorname{rel}\left(B_{i-1}\right)} \text { implies } \\
p\left(t_{1}, \ldots, t_{n}\right) \theta>q\left(s_{1}, \ldots, s_{m}\right) \theta
\end{gathered}
$$

is of the form $u \theta>v \theta$, where $u$ and $v$ are subterms of $p\left(t_{1}, \ldots, t_{n}\right)$, respectively $q\left(s_{1}, \ldots, s_{m}\right)$. We then search for a sequence of terms $u, u_{1}, u_{2}, \ldots, u_{j}, v$, such that for each pair of terms, $u$ and $u_{1}, u_{1}$ and $u_{2}, \ldots, u_{j}$ and $v$, there is a corresponding atom in the sequence $B_{1}, B_{2}, \ldots, B_{i-1}$ that contains both of them.

Assume (without real loss of generality) that $u$ and $u_{1}$ occur in $B_{1}, u_{1}$ and $u_{2}$ occur in $B_{2}, \ldots, u_{j}$ and $v$ occur in $B_{i-1}$. We then select one of these pairs of terms, say $u_{i_{1}}$ and $u_{i_{2}}$ in atom $B_{i_{3}}$, and impose the relations:

$$
\begin{aligned}
& u_{i_{1}}<u_{i_{2}} \text { on } R_{\operatorname{rel}\left(B_{i_{3}}\right)} \text {, and } \\
& u_{i_{1}} \leq u_{i_{2}} \text { on } R_{\operatorname{rel}\left(B_{i_{3}}\right)} \text { for all other pairs of terms and corresponding atoms. }
\end{aligned}
$$

Now we can again use the $T_{P}(M) \subseteq M$ technique to translate such constraints into interargument relations.

Note that this approach involves a search problem: if we fail to verify the proposed inequality constraints, we need to backtrack over the choice of:

- the pair $u_{i_{1}}$ and $u_{i_{2}}$ in $B_{i_{3}}$ with a strict inequality, or

- the sequence of terms $u, u_{1}, u_{2}, \ldots, u_{j}, v$ in $B_{1}, B_{2}, \ldots, B_{i-1}$.

A completely different method for dealing with multiple intermediate bodyatoms is based on the use of unfold/fold steps to group atoms. We illustrate this second method with an example. 
Example 15. The following is the version of the permute program that appeared in $[22]$.

$$
\begin{array}{cc}
\operatorname{perm}([],[]) . & \operatorname{ap} p_{1}([], L, L) . \\
\operatorname{perm}(L,[H \mid T]) \leftarrow & \operatorname{ap}_{1}([H \mid L 1], L 2,[H \mid L 3]) \leftarrow \\
\operatorname{ap}_{2}(V,[H \mid U], L), & \operatorname{ap}_{1}(L 1, L 2, L 3) . \\
\operatorname{ap}_{1}(V, U, W), & \operatorname{ap}_{2}([], L, L) . \\
\operatorname{perm}(W, T) . & \operatorname{ap}_{2}([H \mid L 1], L 2,[H \mid L 3]) \leftarrow \\
& \operatorname{ap}_{2}(L 1, L 2, L 3) .
\end{array}
$$

This example is chosen to illustrate applications of Theorem 3 (the wellmoded case). We would like to prove termination of the goals $\operatorname{perm}\left(t_{1}, t_{2}\right)$, where $t_{1}$ is a ground list and $t_{2}$ a free variable.

Assume the modes perm(in, out), ap $p_{1}$ (in, in, out), ap $p_{2}$ (out, out, in). The orderacceptability imposes, among the others, the following decrease with respect to $>: I \models \operatorname{ap}_{2}(V,[H \mid U], L) \theta \wedge \operatorname{ap}_{1}(V, U, W) \theta$ implies $\operatorname{perm}(L) \theta>\operatorname{perm}(W) \theta$. Note that the underlying decrease $L \theta>W \theta$ cannot be achieved by reasoning on $a p_{1} / 3$ or $\mathrm{ap}_{2} / 3$ alone.

An alternative solution to the one described before is to use the unfold/fold technique to provide a definition for the conjunction of the two intermediate body-atoms. To do this, we start of from a generalised clause, containing the conjunction of atoms both in its head and in its body. In our example we get

$$
\operatorname{ap}_{2}(V,[H \mid U], L), \operatorname{ap}_{1}(V, U, W) \leftarrow \operatorname{ap}_{2}(V,[H \mid U], L), \operatorname{ap}_{1}(V, U, W) .
$$

Next, we unfold both body-atoms, using all applicable clauses, for one resolution step. This gives rise to a generalised program $P^{\prime}$, defining the conjunction of intermediate body-atoms:

$$
\begin{aligned}
& \operatorname{ap}_{2}([],[H \mid T],[H \mid T]), \operatorname{ap}_{1}([], T, T) . \\
& \operatorname{ap}_{2}([H 1 \mid T 1],[H 2 \mid T 2],[H 1 \mid T 3]), \operatorname{ap}_{1}([H 1 \mid T 1], T 2,[H 1 \mid T 4]) \leftarrow \\
& \quad \operatorname{ap}_{2}(T 1,[H 2 \mid T 2], T 3), \operatorname{ap}_{1}(T 1, T 2, T 4) .
\end{aligned}
$$

Now, we need to verify that $M=\left\{\operatorname{ap}_{2}\left(a_{1}, a_{2}, a_{3}\right), a p_{1}\left(b_{1}, b_{2}, b_{3}\right) \mid a_{3}>b_{3}\right\}$ satisfies $T_{P^{\prime}}(M) \subseteq M$. Using the 2 clauses, this is reduced to " $\left[t_{1} \mid t_{2}\right]>t_{2}$ " and " $t_{3}>t_{4}$ implies $\left[t_{5} \mid t_{3}\right]>\left[t_{5} \mid t_{4}\right]$ ", for any terms $t_{1}, t_{2}, t_{3}, t_{4}$ and $t_{5}$, imposing monotonicity and subterm properties on $>$. The proof is completed analogously to the permute example.

It should be noted that in general unfolding can transform a non-terminating program to a terminating one by replacing infinite branches of the LD-tree with failing ones [7]. Bossi and Cocco [7] also stated conditions on unfolding that impose termination to be preserved.

\section{Conclusion}

We have presented a non-transformational approach to termination analysis of logic programs, based on general orderings. The problem of termination was 
studied by a number of authors (see [10] for the survey). More recent work on this topic can be found among others in $[12,13,17,19,23,26,28,30,32]$. The transformational approach to termination has been studied among others in $[1$, $5,18,22,24]$

Our approach gets its power from integrating the traditional notion of acceptability [4] with the wide class of orderings that have been studied in the context of the term-rewriting systems. In theory, such an integration is unnecessary: acceptability (based on level mappings only) is already equivalent to LD-termination. In practice, the required level mappings may sometimes be very complex (such as for Example 1 or Example 12 [15], boolean ring [20] or flattening of a binary tree [5]), and automatic systems for proving termination are unable to generate them. In such cases, generating an appropriate ordering, replacing the level mapping, may often be much easier, especially since we can reuse the impressive machinery on orderings developed for term-rewrite systems. In some other cases, such as turn [8], simple level mappings do exist (in the case of turn: a norm counting the number of 0 s before the first occurrence of 1 in the list is sufficient), but most systems based on level mappings will not even find this level mapping, because they only consider mappings based on term-size or list-length norms. Meta-interpreters, as illustrated in Example 14, give the same complication. Again, our approach is able to deal with such cases.

Sometimes level mappings and norms provide an advantage over general orderings. This is mostly the case if the termination proof can benefit from arguments based on arithmetical operations on the numerical values provided by the level mapping and norm, as illustrated in Example 2. Note however, that general orderings include orderings based on mappings and norms as a special case. We can allow the latter types of orderings as a special case, resorting to them when other orderings in our workbench fail to produce a proof. If we do resort to them, we may allow arithmetic operations on them. The main reason why we defined interargument relations in a very general way is exactly to allow all the power of numerical orderings, and arithmetic, to be applicable in our context.

Unlike transformational approaches, that establish the termination results for logic programs by the reasoning on termination of term-rewriting systems, we apply the orderings directly to the logic programs, thus, avoiding transformations. This could both be regarded as an advantage and as a drawback of our approach. It may be considered as a drawback, because reasoning on successful instances of intermediate body-atoms introduces an additional complication in our approach, for which there is no counterpart in transformational methods (except for the transformation step itself). On the other hand, we consider it as an advantage, because it is precisely this reasoning on intermediate body atoms that gives more insight in the property of logic program termination (as opposed to term-rewrite system termination). Another advantage over transformational approaches is that most of these are restricted to well-moded programs and goals, while our approach does not have this limitation.

So, in a sense our approach provides the best of both worlds: a means to incorporate into 'direct' approaches the generality of general orderings. 
We consider as a future work a full implementation of the approach. Although we already tested very many examples manually, an implementation will allow us to conduct a much more extensive experimentation, comparing the technique also in terms of efficiency with other systems. Since we apply a demand-driven approach, systematically reducing required conditions to more simple constraints on the ordering and the model, we expect that the method can lead to very efficient verification.

\section{Acknowledgements}

We thank Robert Kowalski for continuously stimulating us to look outside of our ivory tower of research to search for challenges in cross-fertilisation of different streams of work.

Alexander Serebrenik is supported by GOA: " $L P^{+}$: a second generation logic programming language". We thank Maurice Bruynooghe for useful suggestions.

\section{References}

1. G. Aguzzi and U. Modigliani. Proving Termination of Logic Program by Transforming them into Equivalent Term Rewriting Systems. In Proc. of 13th Conference on Foundations of Software Technologies and Theoretical Computer Science (FSTE TCS), pages 114-124. Springer Verlag, 1993. LNCS 761.

2. K. R. Apt. From Logic Programming to Prolog. Prentice-Hall Int. Series in Computer Science. Prentice Hall, 1997.

3. K. R. Apt and S. Etalle. On the unification free Prolog programs. In A. M. Borzyszkowski and S. Sokolowski, editors, 18th Int. Symp. on Mathematical Foundations of Computer Science, pages 1-19. Springer Verlag, 1993. LNCS 711.

4. K. R. Apt and D. Pedreschi. Studies in Pure Prolog: Termination. In J. W. Lloyd, editor, Proc. Esprit Symp. on Comp. Logic, pages 150-176. Springer Verlag, 1990.

5. T. Arts. Automatically proving termination and innermost normalisation of term rewriting systems. $\mathrm{PhD}$ thesis, Universiteit Utrecht, 1997.

6. T. Arts and H. Zantema. Termination of logic programs using semantic unification. In M. Proietti, editor, 5th Int. Workshop on Logic Programming Synthesis and Transformation, pages 219-233. Springer Verlag, 1995. LNCS 1048.

7. A. Bossi and N. Cocco. Preserving universal temination through unfold/fold. In G. Levi and M. Rodríguez-Artalejo, editors, Algebraic and Logic Programming, pages 269-286. Springer Verlag, 1994. LNCS 850.

8. A. Bossi, N. Cocco, and M. Fabris. Norms on terms and their use in proving universal termination of a logic program. Theoretical Computer Science, 124(2):297-328, February 1994.

9. F. Bueno, M. J. García de la Banda, and M. V. Hermenegildo. Effectiveness of global analysis in strict independence-based automatic parallelization. In M. Bruynooghe, editor, Logic Programming, Proc. of the 1994 Int. Symp., pages 320-336. MIT Press, 1994.

10. D. De Schreye and S. Decorte. Termination of logic programs: The never-ending story. J. Logic Programming, 19/20:199-260, May/July 1994. 
11. D. De Schreye, K. Verschaetse, and M. Bruynooghe. A framework for analyzing the termination of definite logic programs with respect to call patterns. In I. Staff, editor, Proc. of the Int. Conf. on Fifth Generation Computer Systems., pages 481488. IOS Press, 1992.

12. S. Decorte and D. De Schreye. Termination analysis: some practical properties of the norm and level mapping space. In J. Jaffar, editor, Proc. of the 1998 Joint Int. Conf. and Symp. on Logic Programming, pages 235-249. MIT Press, June 1998.

13. S. Decorte, D. De Schreye, and H. Vandecasteele. Constraint-based termination analysis of logic programs. ACM Transactions on Programming Languages and Systems (TOPLAS), 21(6):1137-1195, November 1999.

14. N. Dershowitz. Termination. In C. Kirchner, editor, First Int. Conf. on Rewriting Techniques and Applications, pages 180-224. Springer Verlag, 1985. LNCS 202.

15. N. Dershowitz and C. Hoot. Topics in termination. In C. Kirchner, editor, Rewriting Techniques and Applications, 5th Int. Conf., pages 198-212. Springer Verlag, 1993. LNCS 690.

16. N. Dershowitz and Z. Manna. Proving termination with multiset orderings. Communications of the ACM (CACM), 22(8):465-476, August 1979.

17. S. Etalle, A. Bossi, and N. Cocco. Termination of well-moded programs. J. Logic Programming, 38(2):243-257, February 1999.

18. H. Ganzinger and U. Waldmann. Termination proofs of well-moded logic programs via conditional rewrite systems. In M. Rusinowitch and J.-L. Remy, editors, Proc. of CTRS'92, pages 216-222. Springer Verlag, 1993. LNCS 656.

19. S. Hoarau. Inférer et compiler la terminaison des programmes logiques avec contraintes. PhD thesis, Université de La Réunion, 1999.

20. J. Hsiang. Rewrite method for theorem proving in first order theory with equality. Journal of Symbolic Computation, 8:133-151, 1987.

21. G. Janssens and M. Bruynooghe. Deriving descriptions of possible values of program variables by means of abstract interpretation. J. Logic Programming, 13(2\&3):205-258, July 1992.

22. M. Krishna Rao, D. Kapur, and R. Shyamasundar. Transformational methodology for proving termination of logic programs. J. Logic Programming, 34:1-41, 1998.

23. N. Lindenstrauss and Y. Sagiv. Automatic termination analysis of logic programs. In L. Naish, editor, Proc. of the Fourteenth Int. Conf. on Logic Programming, pages 63-77. MIT Press, July 1997.

24. M. Marchiori. Logic programs as term rewriting systems. In Proc. of the Algebraic Logic Programming ALP'94, pages 223-241. Springer Verlag, 1994. LNCS; volume 850.

25. L. Plümer. Termination Proofs for Logic Programs. LNAI 446. Springer Verlag, 1990.

26. S. Ruggieri. Verification and validation of logic programs. PhD thesis, Universitá di Pisa, 1999.

27. A. Serebrenik and D. De Schreye. Termination analysis of logic programs using acceptability with general term orders. Technical Report CW 291, Departement Computerwetenschappen, K.U.Leuven, Leuven, Belgium, 2000. Available at http://www.cs.kuleuven.ac.be/publicaties/rapporten/CW2000.html.

28. J.-G. Smaus. Modes and Types in Logic Programming. PhD thesis, University of Kent, 1999.

29. L. Sterling and E. Shapiro. The Art of Prolog. The MIT Press, 1994.

30. C. Taboch. A semantic basis for termination analysis of logic programs. Master's thesis, Ben-Gurion University of the Negev, 1998. 
31. J. D. Ullman and A. van Gelder. Efficient tests for top-down termination of logical rules. Journal of the Association for Computing Machinery, 35(2):345-373, April 1988.

32. S. Verbaeten. Static verification of compositionality and termination for logic programming languages. PhD thesis, Department of Computer Science, K.U.Leuven, Leuven, Belgium, June 2000. v+265+xxvii. 